\title{
Influence of mirror image forces on dispersion and phonon acoustic mode width of quasi-Rayleigh wave interacting with adsorbed atoms
}

\author{
M.Ya. Seneta*, R.M. Peleshchak, V.B. Brytan \\ Drohobych Ivan Franko State Pedagogical University, \\ 3, Stryiska str., 82100 Drohobych, Lviv Region, Ukraine \\ *E-mail: marsen18@i.ua
}

\begin{abstract}
The dispersion law of elastic surface acoustic waves and the energy width of the surface acoustic mode are found to the long-wave approximation depending on the concentration of the adsorbed atoms and the deformation potential of the adatom. The mirror image forces and the nonlocal elastic interaction between the adsorbed atom and the matrix atoms are taken into consideration.
\end{abstract}

Keywords: adatoms, mirror image forces, deformation potential, nonlocal elastic interaction, surface acoustic mode width.

doi: https://doi.org/10.15407/spqeo21.01.041

PACS 81.07.Bc, 66.30.Lw

Manuscript received 19.12.16; revised version received 17.01.18; accepted for publication 29.03.18; published online 29.03.18.

\section{Introduction}

The surface acoustic wave (SAW) method is recently used to diagnose the dynamic properties of twodimensional electronic layers (e.g., the dynamic conductivity, carrier mobility or carrier concentration) of $\mathrm{Al}_{1-\mathrm{x}} \mathrm{Ga}_{\mathrm{x}} \mathrm{As}$ nanoheterostructures [1-3] that have piezoelectric properties. Nanoheterosystems with strained layers $\mathrm{GaAs} / \mathrm{In}_{1-\mathrm{x}} \mathrm{Ga}_{\mathrm{x}} \mathrm{As} / \mathrm{GaAs} \quad$ [4], $\mathrm{Cd}_{1-\mathrm{x}} \mathrm{Zn}_{\mathrm{x}} \mathrm{Te} / \mathrm{CdTe} / \mathrm{Cd}_{1-\mathrm{x}} \mathrm{Zn}_{\mathrm{x}} \mathrm{Te} \quad[5,6], \quad \mathrm{CdTe} / \mathrm{HgTe}$ [6] possess inhomogenious deformation and piezoelectric fields. The surface acoustic wave (SAW) generates the variable electric field and dynamic deformation field. The latter creates roughness on semiconductor surface [7]. This roughness causes appearance of areas on the semiconductor border with the surface electron states, which have different physical properties. These areas are separated by a band gap, the width of which is defined by the height of roughness profile. The height can change depending on the adsorbed atoms concentration and deformation potential [8]. Besides, interaction between the variable electric and dynamic deformation fields and 2D electrons [3], and the adsorbed atoms respectively leads to renormalization of both the velocity and SAW damping coefficient.

In [9], the authors have investigated the influence of interaction between the Rayleigh surface elastic wave and electron-hole plasma of two-dimensional semimetal (HgTe), which is caused by two mechanisms (defor- mation potential and piezoelectric effect), on the dispersion law and Rayleigh wave damping. The influence of structural-distorted isotropic surface layer, grown on the isotropic solid surface, on the dispersion law and the reciprocal Rayleigh length of damping was studied in [10].

The researches of the surface elastic acoustic waves damping processes on the monocrystalline substrate defected surface are topical, because SAW can be a source of long-range effects that induce formation of nanoclusters on the crystal surface beyond the laser irradiation area [11].

The purpose of this paper is to calculate the dispersion law and phonon mode width at different concentrations of adsorbed atoms, considering nonlocal elastic interaction between the adatoms and matrix atoms as well as mirror image forces.

\section{The model. Initial equations and boundary conditions}

Let the semiconductor surface be coinciding with the surface $z=0$, and the axis $z$ is directed from the single crystal surface into the depth. The atomic flux is directed on the semiconductor surface during the molecular beam epitaxy (MBE). Adsorbed atoms could be considered as the surface defects. The subsurface layer with the thickness $a$ ( $a$ - lattice period in $z$-axis direction) is nonuniformly deformed by the adatoms and by the surface elastic acoustic wave due to the deformation potential 
and the local surface energy renormalization. This inhomogeneous self-consistent deformation, in its turn, redistributes the adsorbed atoms across the surface through the deformation potential, i.e., it induces the additional deformation-diffusion flux of adatoms $[12,13]$. Therefore, the influence of the adsorbed atoms reduces to the change of boundary conditions for the stress tensor $\sigma_{i j}$ on the surface $z=0$.

The displacement vector $\vec{u}(\vec{r}, t)$ of points of the medium satisfies the following equation [14]:

$\frac{\partial^{2} \vec{u}}{\partial t^{2}}=c_{t}^{2} \Delta_{\vec{r}} \vec{u}+\left(c_{l}^{2}-c_{t}^{2}\right) \overrightarrow{\operatorname{grad}}(\operatorname{div} \vec{u})$.

The solution of equation (1) for the surface Rayleigh wave propagating along the axis $x$ is:

$$
\begin{aligned}
& u_{x}(x, z)=-i q A e^{i q x-i \omega t-k_{l} z}-i k_{t} B e^{i q x-i \omega t-k_{t} z}, \\
& u_{z}(x, z)=k_{l} A e^{i q x-i \omega t-k_{l} z}+q B e^{i q x-i \omega t-k_{t} z},
\end{aligned}
$$

where $k_{l, t}^{2}=q^{2}-\frac{\omega^{2}}{c_{l, t}^{2}}$; and $A, B$ are the SAW amplitudes.

The direction $x$ on the crystal surface is defined by elastic anisotropy, while on the isotropic surface it could be defined by an external effect, which induces the elastic anisotropy, or due to spontaneous breaking the defect-deformation system, similarly to that in [15].

The deformation $\varepsilon$ on the surface of the semiconductor $(z=0)$ is related with the components of the displacement vector by the relation

$\varepsilon(x, t)=\frac{\partial u_{x}}{\partial x}+\frac{\partial u_{z}}{\partial z}=\frac{\omega^{2}}{c_{l}^{2}} A e^{i q x-i \omega t}$.

Spatially inhomogeneous surface deformation $k_{l, t}^{2} \cdot \varepsilon(x, t)$ leads to the inhomogeneous adatom redistribution $N_{d}(x, t)$ :

$N_{d}(x, t)=N_{d 0}+N_{d 1}(x, t)=N_{d 0}+N_{d 1}(q) e^{i q x-i \omega t}$,

where $N_{d 0}$ is the spatially homogeneous component and $N_{d 1}(q)$ - periodic disturbance amplitude $\left(N_{d 1}<<N_{d 0}\right)$.

The interaction energy between the adsorbed atom and the matrix atoms $W_{d a}$ as an approximation of nonlocal Hooke law $[12,16,17]$ is determined:

$W_{d a}(x)=-\int \lambda\left(\left|x^{\prime}-x\right|\right) \varepsilon\left(x^{\prime}\right) \Delta \Omega_{d} d x^{\prime}$,

where $\lambda$ is the elastic moduli operator [16], and $\Delta \Omega_{d}-$ crystal volume change caused by a single atom adsorbed.

Introducing the variable $\tau=x^{\prime}-x$ and expanding $\varepsilon\left(x^{\prime}\right)$ into a Taylor series, we obtain:

$$
\begin{aligned}
& W_{d a}^{\text {int }}(x)=-\int \lambda(|\tau|) \varepsilon(x+\tau) \Delta \Omega_{d} d \tau= \\
& -\int \lambda(|\tau|)\left(\varepsilon(x)+\frac{\partial^{2} \varepsilon(x)}{\partial x^{2}} \cdot \frac{\tau^{2}}{2}\right) \Delta \Omega_{d} d \tau= \\
& -K_{d} \varepsilon(x) \Delta \Omega_{d}-K_{d} \frac{\partial^{2} \varepsilon(x)}{\partial x^{2}} l_{d}^{2} \Delta \Omega_{d},
\end{aligned}
$$

where $K_{d}=\int \lambda(|\tau|) d \tau \equiv K$ is the modulus of elasticity, and $l_{d}^{2}=\frac{\int \lambda(\tau) \tau^{2} d \tau}{2 \int \lambda(|\tau|) d \tau}-$ average of the square of characteristic distance of interaction between the adatom and matrix atoms.

The adsorbed atoms elastic fields shift the atoms in the neighborhood of other adatoms, thus creating the forces that affect them, and cause the elastic interaction between them. The energy of this interaction decreases in accordance with the power law and is rather significant, when the crystal lattice is heavily deformed by the adatoms. Within isotropic solids, the elastic defect interaction energy equals to zero.

The adsorbed atoms elastic interaction decreases according to the power law, as the distance increases. Besides, there is another interaction that changes gradually at distances comparable to the crystal size and is connected with mirror image forces applied to the crystal surface [17], which provide boundary conditions on the crystal surface $(z=0)$ (e.g., the condition that there are no stresses on the crystal surface). The field of these forces is called the image field or the imaginary sources field, like to the electrostatic field of charge arising on the conductive surface, and is equivalent to the mirror image charge field [18]. The interaction energy $W_{d d}^{\text {int }}$ between the adsorbed atom in the position $r^{\prime}$ and other adatoms with the concentration $N_{d}(x)$ is caused by these forces, is virtually independent of the adatom position $r^{\prime}$ and can be defined as that in [17]:

$W_{d d}^{\mathrm{int}}(x)=-\frac{2}{3} \frac{1-2 \mathrm{v}}{K(1-\mathrm{v}) a} \theta_{s}^{2} N_{d}(x)$,

where $v$ is the Poisson coefficient; $\theta_{s}=K \cdot \Delta \Omega_{d}$ - surface deformation potential.

The force $F$ acting on the adatom due to the elastic field appearing in the implanted adatoms matrix is given by:

$$
\begin{aligned}
& F=-\frac{\partial\left(W_{d d}^{\mathrm{int}}(x)+W_{d a}^{\mathrm{int}}(x)\right)}{\partial x}= \\
& -\frac{2}{3} \frac{1-2 v}{K(1-v) a} \theta_{s}^{2} \frac{\partial N_{d}(x)}{\partial x}+\theta_{s} \frac{\partial \varepsilon(x, t)}{\partial x}+\theta_{s} l_{d a}^{2} \frac{\partial^{3} \varepsilon(x, t)}{\partial x^{3}}
\end{aligned}
$$

which induces the regular diffusion flux $\left(-D_{d} \frac{\partial N_{d}(x)}{\partial x}\right)$ and the additional deformation flux of adatoms. The latter is caused by the deformation gradients $\frac{\partial \varepsilon(x, t)}{\partial x}$ and the defect concentration one $\frac{\partial N_{d}(x)}{\partial x}$.

Analysis of the formula (9) shows that the concentration gradient $\left(-\theta_{s}^{2} \partial N_{d}(x) / \partial x\right)$ creates a deformation flux component directed to the side where the adatom concentration is higher (the first term), unlike the regular diffusion flux. Furthermore, the adatoms that are the extension centers $\left(\Delta \Omega_{d}>0\right)$ will move to the area 
that experiences the tensile strain, while the adatoms that are the compression centers $\left(\Delta \Omega_{d}<0\right)$ will move to the area of the relative compression (the second term).

Under the force (9), the adatoms in the elastic field aquire the velocity

$$
\begin{gathered}
v=\mu \cdot F=\frac{2}{3} \frac{1-2 v}{K(1-v)} \frac{D_{d} \theta_{s}^{2}}{k_{\mathrm{B}} T a} \frac{\partial N_{d}(x)}{\partial x}+ \\
+\frac{D_{d} \theta_{s}}{k_{\mathrm{B}} T} \frac{\partial \varepsilon(x, t)}{\partial x}+\frac{D_{d} \theta_{s}}{k_{\mathrm{B}} T} l_{d a}^{2} \frac{\partial^{3} \varepsilon(x, t)}{\partial x^{3}},
\end{gathered}
$$

where $D_{d}$ is the adatom diffusion coefficient, $T$ temperature, $k_{\mathrm{B}}-$ Boltzmann constant. Here, the Einstein relation is used to determine the mobility of adatoms $\mu$.

Taking into account (10) and the continuity equation $\left(\operatorname{div} \vec{j}=-\frac{\partial N_{d}(x, t)}{\partial t}\right)$, the flux of implanted adatoms and the equation for the concentration of adatoms can be written as follows:

$$
\begin{aligned}
& j=-D_{d} \frac{\partial N_{d}(x, t)}{\partial x}+ \\
& +\frac{D_{d} \theta_{s}}{k_{\mathrm{B}} T} \cdot N_{d}(x, t) \frac{\partial}{\partial x}\left(\begin{array}{l}
\frac{2}{3} \frac{1-2 v}{K(1-v) a} \theta_{s} N_{d}(x, t)+ \\
+\varepsilon(x, t)+l_{d a}^{2} \frac{\partial^{2} \varepsilon(x, t)}{\partial x^{2}}
\end{array}\right), \\
& \frac{\partial N_{d}(x, t)}{\partial t}=D_{d} \frac{\partial^{2} N_{d}(x, t)}{\partial x^{2}}- \\
& -\frac{D_{d} \theta_{s}}{k_{\mathrm{B}} T} \cdot \frac{\partial}{\partial x}\left(N_{d}(x, t) \frac{\partial}{\partial x}\left(\begin{array}{l}
\frac{2}{3} \frac{1-2 v}{K(1-v) a} \theta_{s} N_{d}(x, t)+ \\
+\varepsilon(x, t)+l_{d a}^{2} \frac{\partial^{2} \varepsilon(x, t)}{\partial x^{2}}
\end{array}\right)\right),
\end{aligned}
$$

The first term in (12) describes the regular gradient concentration diffusion, while the second one describes a qualitatively new diffusion effect of the "flow of deformation retraction" caused by mirror image forces and the deformation gradient [19] as well as the nonlocal interaction between the adatoms and the surface atoms [20].

Considering the condition $N_{d 1} \ll<N_{d 0}$ and (5), the equation (12) in a linear approximation will be:

$$
\begin{aligned}
& \left(-i \omega+D_{d}\left(1-\frac{2}{3} \frac{1-2 v}{K(1-v) a} \frac{\theta_{d}^{2}}{k T} N_{d 0}\right) q^{2}\right) N_{1 d}(q)= \\
& =\frac{D_{d} \theta_{d}}{k T} N_{d 0} \varepsilon(q) q^{2}\left(1-l_{d}^{2} q^{2}\right) .
\end{aligned}
$$

From Eq. (13), we obtain the expression for the amplitude of the surface adatom concentration $N_{d 1}(q)$.

The spatially inhomogeneous adatom distribution modulates the surface energy

$$
F(x)=F_{0}+\frac{\partial F}{\partial N_{d 1}} \cdot N_{d 1}(x),
$$

which results in the lateral mechanical stress $\sigma_{x z}=\frac{\partial F(N(x))}{\partial x}$,

that is compensated by the displacement stress within the medium [14]. The boundary condition expressing the balance of lateral stresses is as follows:

$$
\left.\mu\left(\frac{\partial u_{x}}{\partial z}+\frac{\partial u_{z}}{\partial x}\right)\right|_{z=0}=\left(\frac{\partial F}{\partial N_{d 1}}\right) \frac{\partial N_{d 1}(x)}{\partial x}
$$

where $\mu$ is the displacement module of the medium.

The coefficient $\frac{\partial F}{\partial N_{d 1}}$ is considered by us as a predetermined phenomenological parameter.

In addition, interaction between the adatoms and semiconductor atoms results in a normal mechanical stress on the surface. The corresponding boundary condition has the form:

$\left.\left(\frac{\partial u_{z}}{\partial z}+(1-2 \beta) \frac{\partial u_{x}}{\partial x}\right)\right|_{z=0}=\frac{\theta_{d} N_{d 1}(x)}{\rho c_{l}^{2} a}$,

where $a$ is the crystal lattice parameter on the semiconductor surface, $\beta=\frac{c_{t}^{2}}{c_{l}^{2}}, \rho-$ crystal density.

\section{The dispersion equation and the phonon mode width of the surface acoustic wave interacting with adsorbed atoms}

The dispersion equation and the phonon mode width are obtained from the boundary conditions (14), (15) that express the balance of lateral and normal stresses on the semiconductor surface. We substitute (13), taking into account (4) and (5) as well as (2) and (3), into (14) and (15). As a result, we obtain a system of two linear equations for the amplitudes $A$ and $B$. Then, from the condition of nontrivial solutions we obtain: $\left(q^{2}+k_{t}^{2}\right)^{2}-4 q^{2} k_{l} k_{t}=-\frac{2}{\beta} \frac{\omega^{2}}{c_{l}^{2}} \frac{\theta_{d}}{k T} \frac{N_{d 0}}{\rho c_{l}^{2}} \times$

$$
\begin{gathered}
\times \frac{D_{d} q^{2}}{-i \omega+D_{d}\left(1-\frac{2}{3} \frac{1-2 v}{K(1-v) a} \frac{\theta_{d}^{2}}{k T} N_{d 0}\right) q^{2}} \times \\
\times\left(1-l_{d}^{2} q^{2}\right) \cdot\left(q^{2} k_{t} \frac{\partial F}{\partial N_{d 1}}+\left(q^{2}+k_{t}^{2}\right) \frac{\theta_{d}}{2 a}\right) .
\end{gathered}
$$

The left part of (16) matches the Rayleigh determinant; the latter, when being equal to zero, determines the dispersion law of the Rayleigh surface acoustic wave without adsorbed atoms [14]. The right part of (16) renormalizes the dispersion equation of the Rayleigh acoustic wave due to the adsorbed atoms power action $\left(\sim \theta_{d}\right)$. The adsorbed atoms deform the subsurface layer in the crystal lattice. Substituting $\omega=c_{t} q \xi$ into (16), one can obtain: 


$$
\begin{aligned}
& \left(2-\xi^{2}\right)^{2}-4 \sqrt{1-\xi^{2}} \sqrt{1-\frac{c_{t}^{2}}{c_{l}^{2}} \xi^{2}}=-\frac{2 \xi^{2} \theta_{d} N_{d 0}}{k T \rho c_{l}^{2}} \times \\
& \times \frac{D_{d} q\left(D_{d}\left(1-\frac{2}{3} \frac{1-2 v}{K(1-v) a} \frac{\theta_{d}^{2}}{k T} N_{d 0}\right) q+i c_{t} \xi\right)}{\left(D_{d}\left(1-\frac{2}{3} \frac{1-2 v}{K(1-v) a} \frac{\theta_{d}^{2}}{k T} N_{d 0}\right)\right)^{2} q^{2}+c_{t}^{2} \xi^{2}} \times \\
& \times\left(1-l_{d}^{2} q^{2}\right) \cdot\left(q^{2} \sqrt{1-\xi^{2}} \frac{\partial F}{\partial N_{d 1}}+\left(2-\xi^{2}\right) \frac{\theta_{d}}{2 a}\right) .
\end{aligned}
$$

Eq. (17) has the real part and the imaginary one that finally determine a correction to the dispersion law of Rayleigh wave and its damping. The multiplier $q$ in the numerator of (17) allows to solve this equation using the iterative method in the long-wave region $q a<<1$.

Let the left part of (17) be denoted by the function $f(\xi)$, and let us to expand the latter into a Taylor series in the vicinity of the point $\xi_{0}$ :

$f\left(\xi_{0}+\delta \xi\right) \approx f\left(\xi_{0}\right)+f^{\prime}\left(\xi_{0}\right) \delta \xi$,

where $\xi_{0}$ is the solution of the equation $f\left(\xi_{0}\right)=0$.

Then, the correction $\delta \xi$ is determined by the right part of (17) with the substitution $\xi \rightarrow \xi_{0}$.

$$
\begin{aligned}
& \delta \xi=\frac{1}{f^{\prime}\left(\xi_{0}\right)} \frac{2 \xi_{0}^{2} \theta_{d} N_{d 0}}{k T \rho c_{l}^{2}} \times \\
& \times \frac{D_{d}^{2} q^{2}\left(1-\frac{2}{3} \frac{1-2 v}{K(1-v) a} \frac{\theta_{d}^{2}}{k T} N_{d 0}\right)}{\left(D_{d}\left(1-\frac{2}{3} \frac{1-2 v}{K(1-v) a} \frac{\theta_{d}^{2}}{k T} N_{d 0}\right)\right)^{2} q^{2}+c_{t}^{2} \xi_{0}^{2}} \times \\
& \times\left(1-l_{d}^{2} q^{2}\right) \cdot\left(q \sqrt{1-\xi_{0}^{2}} \frac{\partial F}{\partial N_{d 1}}+\left(2-\xi_{0}^{2}\right) \frac{\theta_{d}}{2 a}\right)- \\
& -i \frac{1}{f^{\prime}\left(\xi_{0}\right)} \frac{2 c_{t} \xi_{0}^{3} \theta_{d} N_{d 0}}{k T \rho c_{l}^{2}} \times \\
& \times \frac{D_{d} q}{\left(D_{d}\left(1-\frac{2}{3} \frac{1-2 v}{K(1-v) a} \frac{\theta_{d}^{2}}{k T} N_{d 0}\right)\right)^{2} q^{2}+c_{t}^{2 \xi_{0}^{2}}} \times \\
& \times\left(1-l_{d}^{2} q^{2}\right)\left(q \sqrt{1-\xi_{0}^{2}} \frac{\partial F}{\partial N_{d 1}}+\left(2-\xi_{0}^{2}\right) \frac{\theta_{d}}{2 a}\right) .
\end{aligned}
$$

The numerical analysis shows that $f^{\prime}\left(\xi_{0}\right)>0$ in the whole region of changing $\xi_{0}$.

Separating the real and imaginary parts in (19) and considering $\omega=c_{t} q \xi_{0}+c_{t} q \delta \xi$, we obtain the expressions for the dispersion law $\omega^{\prime}(q)$ of the elastic surface acoustic wave and its width $\omega^{\prime \prime}(q)$, which is caused by the interaction between the adsorbed atoms and the self-consistent quasi-Rayleigh wave in consideration of both the nonlocal elastic interaction of implanted impurity with the matrix atoms [16], and the mirror image forces [17]:

$$
\begin{aligned}
& \omega^{\prime}(q)=c_{t} q \xi_{0} \times \\
& \left(1-\frac{1}{f^{\prime}\left(\xi_{0}\right)} \frac{2 \xi_{0} \theta_{d} N_{d 0}}{k T \rho c_{l}^{2}} \times\right. \\
& \times\left(\begin{array}{l}
\times \frac{D_{d}^{2} q^{2}\left(1-\frac{2}{3} \frac{1-2 v}{K(1-v) a} \frac{\theta_{d}^{2}}{k T} N_{d 0}\right)}{\left(D_{d}\left(1-\frac{2}{3} \frac{1-2 v}{K(1-v) a} \frac{\theta_{d}^{2}}{k T} N_{d 0}\right)\right)^{2} q^{2}+c_{t}^{2 \xi_{0}^{2}}} \times \\
\times\left(1-l_{d}^{2} q^{2}\right) \cdot\left(q \sqrt{1-\xi_{0}^{2}} \frac{\partial F}{\partial N_{d 1}}+\left(2-\xi_{0}^{2}\right) \frac{\theta_{d}}{2 a}\right)
\end{array}\right) \\
& \omega^{\prime \prime}(q)=-c_{t}^{2} \frac{1}{f^{\prime}\left(\xi_{0}\right)} \frac{2 \xi_{0}^{3} \theta_{d} N_{d 0}}{k T \rho c_{l}^{2}} \times \\
& \times \frac{D_{d} q^{2}}{\left(D_{d}\left(1-\frac{2}{3} \frac{1-2 v}{K(1-v) a} \frac{\theta_{d}^{2}}{k T} N_{d 0}\right)\right)^{2} q^{2}+c_{t}^{2} \xi_{0}^{2}} \times \\
& \times\left(1-l_{d}^{2} q^{2}\right) \cdot\left(q \sqrt{1-\xi_{0}^{2}} \frac{\partial F}{\partial N_{d 1}}+\left(2-\xi_{0}^{2}\right) \frac{\theta_{d}}{2 a}\right) .
\end{aligned}
$$

\section{Numerical calculation and analysis of mirror image forces influence on the dispersion and surface elastic acoustic mode width}

Calculation of the elastic SAW dispersion law dependence $\omega^{\prime}(q)=\operatorname{Re} \omega(q)$ and the acoustic mode width $\omega^{\prime \prime}(q)=\operatorname{Im} \omega(q)$ has been performed for the GaAs semiconductor with the adsorbed atom surface concentration $N_{d 0}=3 \cdot 10^{12} \mathrm{~cm}^{-2}, 3 \cdot 10^{13} \mathrm{~cm}^{-2}$ at the

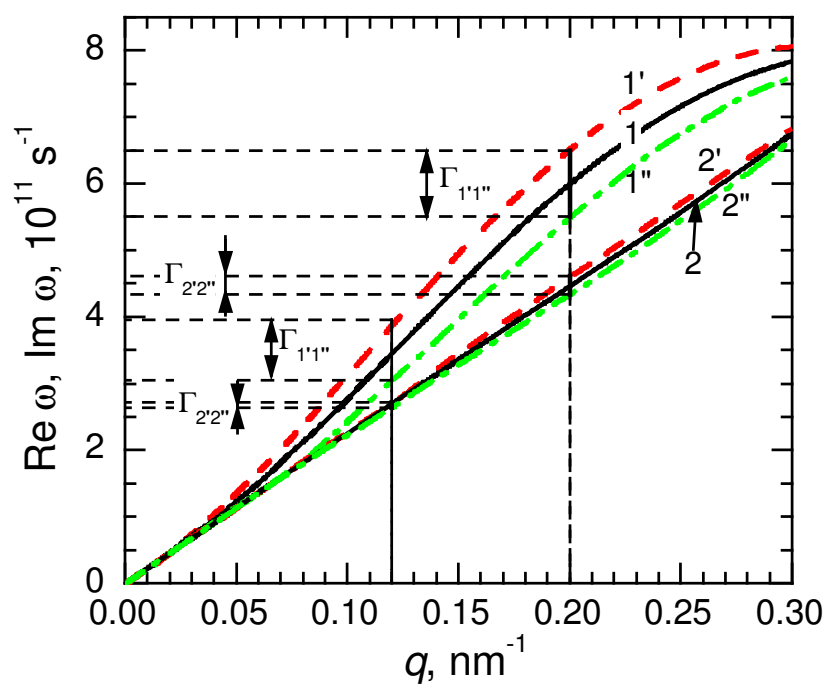

Fig. 1. The dispersion law $\omega^{\prime}(q)(1,2)$ and the phonon mode width $\omega^{\prime \prime}(q)\left(1^{\prime}, 1^{\prime \prime}, 2^{\prime}, 2^{\prime \prime}\right)$ of the surface elastic acoustic wave interacting with the adsorbed atoms with considering the mirror image forces and nonlocal elastic interaction $\left(l_{d} \neq 0\right)$ between the adatoms and the matrix atoms at the values of adatoms concentration $N_{d 0}=3 \cdot 10^{13} \mathrm{~cm}^{-2}\left(1, l^{\prime}, l^{\prime \prime}\right) ; N_{d 0}=3 \cdot 10^{12} \mathrm{~cm}^{-2}(2$, $\left.2^{\prime}, 2^{\prime \prime}\right)$. 
following parameter values: $l_{d}=2.9 \mathrm{~nm}, a=0.565 \mathrm{~nm}$, $c_{l}=4400 \mathrm{~m} / \mathrm{s}, c_{t}=2475 \mathrm{~m} / \mathrm{s}, \rho=5320 \mathrm{~kg} / \mathrm{m}^{3}, \theta_{d}=10 \mathrm{eV}$, $D_{d}=5 \cdot 10^{-2} \mathrm{~cm}^{2} / \mathrm{s}, \frac{\partial F}{\partial N_{d 1}}=0.1 \mathrm{eV}, T=100 \mathrm{~K}[13]$.

The characteristic length value $l_{d}$ of interaction between the adatom and lattice atoms has been found from the condition of free energy minimum of the crystal with adsorbed atoms [21].

Fig. 1 shows the calculation results of the dispersion law $\omega^{\prime}(q)$ (Fig. 1, curves 1,2) of the surface elastic acoustic mode and its width $\omega^{\prime \prime}(q)$ dependence on the module of wave vector $q$ (Fig. 1, curves $1^{\prime}, 1^{\prime \prime}, 2^{\prime}, 2^{\prime \prime}$ ), caused by interaction between the adsorbed atoms and self-consistent acoustic quasi-Rayleigh wave at two values of the adsorbed atoms concentration $N_{d 0}$ with considering the mirror image forces.

For $q \rightarrow 0$, the surface acoustic mode width $\omega^{\prime \prime}(q)$ tends to zero, while the dispersion curve $\omega^{\prime}(q)$ approaches asymptotically to the surface Rayleigh wave dispersion curve according to the dispersion law $\omega(q)=c_{t} \xi_{0} q$. It should be noted that at $q=1 / l_{d}$ the surface acoustic wave length is the same as the characteristic length of the adatom interaction with the lattice atoms. Fig. 1 shows that the acoustic phonon mode width increases as the adsorbed atoms concentration increases.

In particular, at $q=0.12 \mathrm{~nm}^{-1}$ and the adatoms concentrations $N_{d 0}=3 \cdot 10^{12} \mathrm{~cm}^{-2}$ the energy width $\Gamma_{2^{\prime} 2^{\prime \prime}}=$ $\hbar \omega^{\prime \prime}(q)$ of the surface acoustic mode is equal to $9 \mu \mathrm{eV}$, and at $N_{d 0}=3 \cdot 10^{13} \mathrm{~cm}^{-2}$ the energy width $\Gamma_{1^{\prime} 1^{\prime \prime}}$ is equal to $55 \mu \mathrm{eV}$, while in more short-wave region of elastic surface acoustic waves $\left(q=0.2 \mathrm{~nm}^{-1}\right)$ at the concentrations $N_{d 0}=3 \cdot 10^{12} \mathrm{~cm}^{-2}$ and $3 \cdot 10^{13} \mathrm{~cm}^{-2}$ the energy width is equal to 17 and $67 \mu \mathrm{eV}$, respectively.

Fig. 2 shows the dispersion dependences $\omega^{\prime}(q)$ and the energy widths $\omega^{\prime \prime}(q)$ of the surface acoustic modes at the adatom concentration $N_{d 0}=3 \cdot 10^{13} \mathrm{~cm}^{-2}$ with (Fig. 2, curves $1,1^{\prime}, 1^{\prime \prime}$ ) and without (Fig. 2, curves 2, 2', 2") considering the mirror image forces. In particular, at $q=$ $0.12 \mathrm{~nm}^{-1}, 0.2 \mathrm{~nm}^{-1}$ the energy widths of surface acoustic modes without considering the mirror image forces are 89 and $167 \mu \mathrm{eV}$, respectively. From the comparative analysis of the energy widths of acoustic modes we obtain that the mirror image forces at $q=0.12 \mathrm{~nm}^{-1}$ reduce the surface acoustic mode energy width by $34 \mu \mathrm{eV}$, while at $q=0.2 \mathrm{~nm}^{-1}$ the reduction is by $101 \mu \mathrm{eV}$.

If the nonlocal elastic interaction between the adatoms and the matrix atoms is not considered $\left(l_{d}=0\right)$ (Fig. 3), at $q=0.12 \mathrm{~nm}^{-1}$ and the adatom concentration $N_{d 0}=3 \cdot 10^{12} \mathrm{~cm}^{-2}$ the energy width $\Gamma_{2} 2^{\prime \prime}$ is equal to $28 \mu \mathrm{eV}$, and at $N_{d 0}=3 \cdot 10^{13} \mathrm{~cm}^{-2} \Gamma_{1^{\prime} 1 "}=100 \mu \mathrm{eV}$.

Fig. 4 shows the dispersion dependences $\omega^{\prime}(q)$ and the energy widths $\omega^{\prime \prime}(q)$ of the surface acoustic modes with (Fig. 4, curves $1,1^{\prime}, 1^{\prime}$ ) and without (Fig. 4, curves $\left.2,2^{\prime}, 2^{\prime \prime}\right)$ considering the mirror image forces at the adatom concentration $N_{d 0}=3 \cdot 10^{13} \mathrm{~cm}^{-2}$. In particular, at $q=0.2 \mathrm{~nm}^{-1}$ the energy width of surface acoustic modes without considering the mirror image forces is equal to $251 \mu \mathrm{eV}$. From the comparative results, we can see that

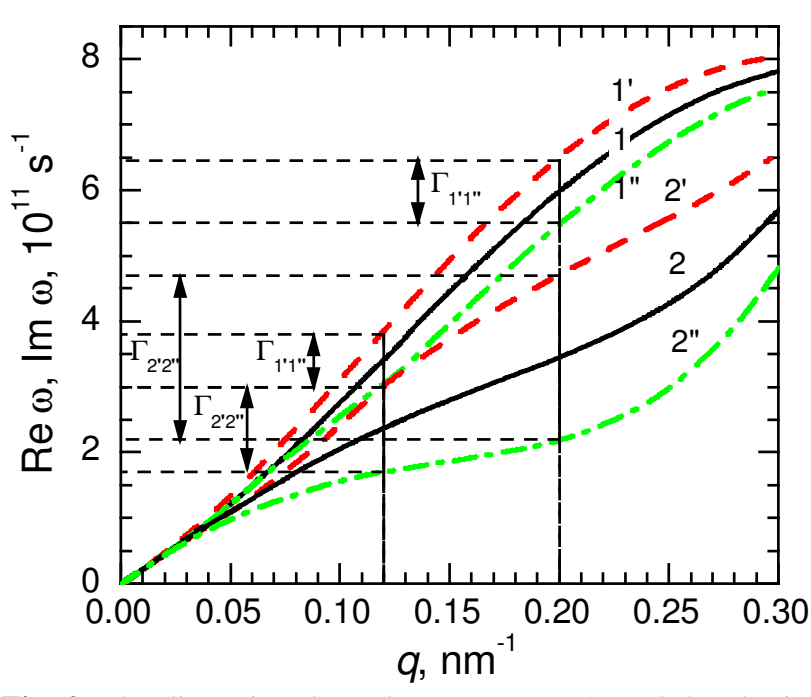

Fig. 2. The dispersion dependence $\omega^{\prime}(q)(1,2)$ and the elastic surface phonon mode width $\omega^{\prime \prime}(q)\left(1^{\prime}, 1^{\prime \prime} ; 2^{\prime}, 2^{\prime \prime}\right)$ interacting with adsorbed atoms with $\left(1,1^{\prime}, 1^{\prime \prime}\right)$ and without $\left(2,2^{\prime}, 2^{\prime \prime}\right)$ considering the mirror image forces at the value of adsorbed atoms concentration $N_{d 0}=3 \cdot 10^{13} \mathrm{~cm}^{-2}$.

the mirror image forces reduce the surface acoustic mode energy width by $151 \mu \mathrm{eV}$.

From the comparative analysis of the dispersion law $\omega^{\prime}(q)$ and the phonon mode width of the surface elastic acoustic wave, we obtain that in the acoustic mode shortwave region the influence of the mirror image forces on the change of the surface acoustic mode energy width is more significant. If the nonlocal interaction is not considered $\left(l_{d}=0\right)$, the phase velocity of quasi-Rayleigh wave emission $\left(v_{p h}=\omega(q) / q\right)$ is higher than in the case when this interaction is considered.

Practically, SAW can be used as optical hologram reading transmitters in photorefractive crystals [22]. The change of Raman spectra energy position of maximums

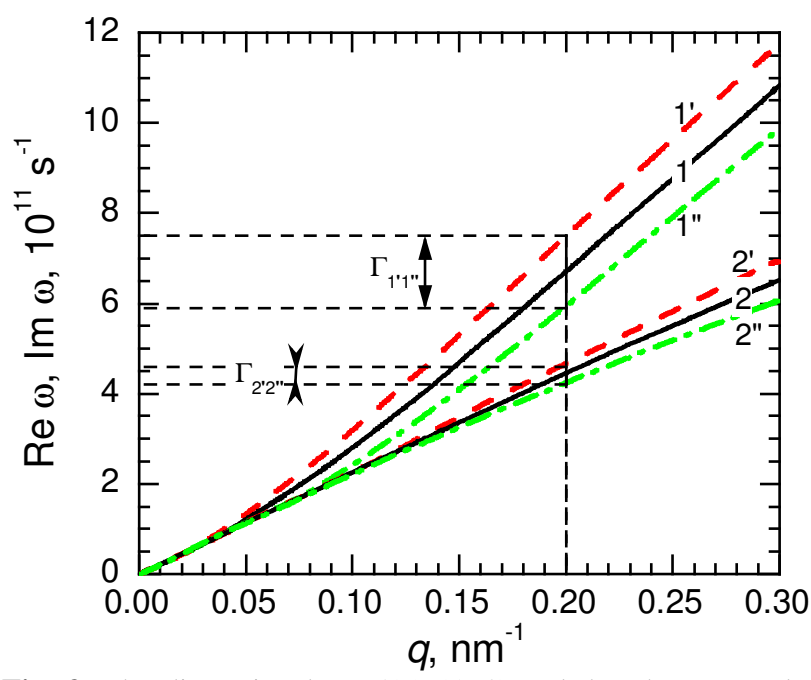

Fig. 3. The dispersion law $\omega^{\prime}(q)(1,2)$ and the phonon mode width $\omega^{\prime \prime}(q)\left(1^{\prime}, 1^{\prime \prime} ; 2^{\prime}, 2^{\prime \prime}\right)$ of the surface elastic acoustic wave interacting with the adsorbed atoms without considering the nonlocal elastic interaction $\left(l_{d}=0\right)$ between the adatoms and the matrix atoms and with considering the mirror image forces at the values of adatoms concentration $N_{d 0}=3 \cdot 10^{13} \mathrm{~cm}^{-2}\left(1,1^{\prime}\right.$, $\left.1^{\prime \prime}\right) ; 3 \cdot 10^{12} \mathrm{~cm}^{-2}\left(2,2^{\prime}, 2^{\prime \prime}\right)$. 


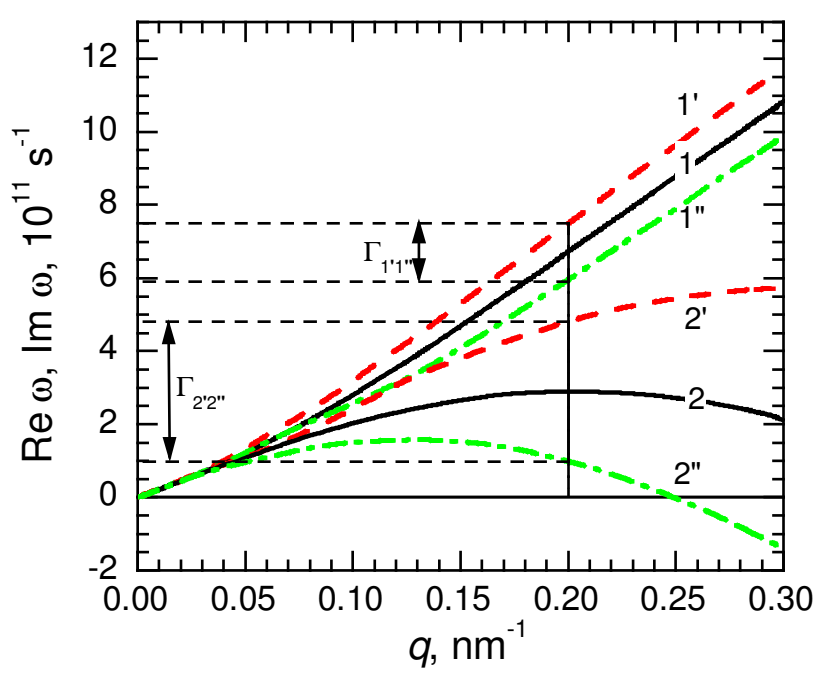

Fig. 4. The dispersion dependence $\omega^{\prime}(q)(1,2)$ and the elastic surface phonon mode width $\omega^{\prime \prime}(q)\left(1^{\prime}, 1^{\prime \prime} ; 2^{\prime}, 2^{\prime \prime}\right)$ interacting with adsorbed atoms with $\left(1,1^{\prime}, 1^{\prime \prime}\right)$ and without $\left(2,2^{\prime}, 2^{\prime \prime}\right)$ considering the mirror image forces, and without considering the nonlocal elastic interaction $\left(l_{d}=0\right)$ between the adatoms and the matrix atoms at the value of adsorbed atoms concentration $N_{d 0}=3 \cdot 10^{13} \mathrm{~cm}^{-2}$.

is caused by the nonelastic light scattering (MandelstamBrillouin). Moreover, the experimental data of this change, depending on the adsorbed atoms concentration and the strain potential, can be used in the selective gas sensors construction on the elastic surface acoustic modes [23].

\section{Conclusions}

1. The elastic surface acoustic wave dispersion theory depending on the adsorbed atom concentration is developed within the nonlocal elastic interaction between the adsorbed atoms and the matrix atoms in consideration of the mirror image forces.

2. We have established that:

a) the mirror image forces reduce the surface elastic acoustic mode energy width, and in the shortwave region of acoustic mode the influence of the mirror image forces on the change of the surface acoustic mode energy width is more significant;

b) the nonlocal elastic interaction influences on the phase velocity of quasi-Rayleigh wave emission. The phase velocity is higher in the case when nonlocal elastic interaction is not considered. Moreover, this nonlocal elastic interaction leads to reduction of the surface elastic acoustic mode energy width. The influence is more significant in the short-wave region of acoustic mode;

c) the surface acoustic mode energy width is proportional to the product of the adsorbed atoms concentration and the adsorbed atom surface deformation potential;

d) at the same concentrations of the adsorbed atoms located in the subsurface crystal lattice as the interstitial impurities, the surface acoustic mode width is higher than in the case when the adsorbed atoms are the substitutional impurities.

\section{References}

1. Wixforth A., Kotthaus J.P., and Weimann G. Quantum oscillations in the surface-acoustic-wave attenuation caused by a two-dimensional electron system. Phys. Rev. Lett. 1986. 56, No 19. P. 2104.

2. Willett R.L., West K.W., and Pfeiffer L.N. Apparent inconsistency of observed composite fermion geometric resonances and measured effective mass. Phys. Rev. Lett. 1995. 75, No 16. P. 2988.

3. Fil' D.V. Interaction of nonuniform elastic waves with two-dimensional electrons in AlGaAs-GaAsAlGaAs heterostructures. Low Temp. Phys. 1999. 25. P. 466; doi: 10.1063/1.593768.

4. Karpovich I.A., Anshon A.V., Filatov D.O. Defect generation and passivation in the strained quantum well $\mathrm{GaAs} / \mathrm{InGaAs}$ heterostructures under hydrogen plasma treatment. Semiconductors. 1998. 32, No 9. P. 1089.

5. Kim T.W., Park H.L. Interband transition and electronic subband studies in CdTe/ZnTe strained single and double quantum wells grown by doublewell temperature-gradient vapor deposition. $J$. Cryst. Growth. 1996. 159. P. 467-470; doi: 10.1016/0022-0248(95)00636-2.

6. Kozlovsky V.I., Litvinov V.G., Sadofyev Yu.G. Band offset in $\mathrm{Zn}_{1-\mathrm{x}} \mathrm{Cd}_{\mathrm{x}} \mathrm{Te} / \mathrm{ZnTe}$ single quantum well structure grown by molecular beam epitaxy on GaAs (001). Semiconductors. 2000. 34, No 8. P. 998.

7. Khankina S.I., Yakovenko V.M., Yakovenko I.V. Surface electron states produced by a Rayleigh wave. J. Exp. Theor. Phys. 2007. 104, No 3. P. 467; doi: 10.1134/S1063776107030132.

8. Seneta M.Ya., Peleschak O.R., Peleshchak R.M., Uhryn Yu.O. in: Proc. IX Intern. Conf. on Topical Problems of Semiconductor Physics, Truskavets, 2016, ed. by R. Pazyuk (Publ. House "UKRPOL" Ltd., Drohobych), P. 152.

9. Kovalev V.M, Chaplik A.V. Interaction of surface and bulk acoustic waves with a twodimensional semimetal. J. Exp. Theor. Phys. 2015. 120, No 2. P. 312-318; doi: 10.1134/ S1063776115020028.

10. Kosachev V.V., Gandurin Y.N., Murav'ev S.E. Effect of the structurally damaged surface layer of an isotropic solid on Rayleigh wave dispersion and damping. Phys. Solid State. 2011. 53, No 10. P. 2174; doi: 10.1134/S1063783411100179

11. Vlasenko A.I., Baidullaeva A., Veleschuk V.P., Mozol P.E., Boiko N.I., Litvin O.S. On the formation of nanostructures on a CdTe surface, stimulated by surface acoustic waves under nanosecond laser irradiation. Semiconductors. 2015. 49, No 2. P. 229 ; doi: 10.1134/S1063782615020220.

12. Peleshchak R.M., Kuzyk O.V., Dan'kiv O.O. Temperature regimes of formation of nanometer periodic structure of adsorbed atoms in GaAs semiconductors under the action of laser irradiation. 
Condens. Matter Phys. 2015. 18, No 4. P. 43801; doi: 10.5488/CMP.18.43801.

13. Peleshchak R.M., Lazurchak I., Kuzyk O.V., Dan'kiv O.O., Zegrya G.G. Role of acoustoelectric interaction in the formation of nanoscale periodic structures of adsorbed atoms. Semiconductors. 2016. 50, No 3. P. 314; doi: 10.1134/S1063782616030180.

14. Landau L.D., Lifshitz E.M. Theory of Elasticity. Pergamon Press, London, 1970. P. 165.

15. Haken H. Synergetics. Springer, 1983.

16. Kunin I.A. Nonlocal Theory of Elasticity. Moscow, Nauka, 1975 (in Russian).

17. Krivoglaz M.Ya. Diffraction of X-rays and Neutrons in Imperfect Crystals. Kiev, Naukova Dumka, 1983 (in Russian).

18. Kosevich A.M. The Theory of Crystal Lattice. Kharkov, 1988 (in Russian).

19. Falco G.L., Shpyrko S.G. Model of strain redistribution and concentration stationary profiles of implanted impurity. Ukr. J. Phys. 1995. 40, No 10. P. 1115.

20. Emel'yanov V.I., Eremin K.I. Threshold nucleation of a nanometer-scale periodic adatom structure with the participation of a static surface acoustic wave. JETP Lett. 2002. 75, No 2. P. 98; doi: 10.1134/1.1466485.

21. Peleshchak R.M., Kuzyk O.V., Dan'kiv O.O. Diffusion-deformation theory of the formation of self-assembled nanoclusters of the implanted impurities. J. Phys. Stud. 2013. 17, No 2. P. 2601.

22. Deev V.N., Pyatakov P.A. Optical generation of acoustic waves on photorefractive lattice at pulsating lighting. Tech. Phys. Lett. 1990. 60, No 1. P. 91.

23. Wagner J., Ramsteiner M., Wild Ch., Koidl P. Resonant Raman scattering of amorphous carbon and polycrystalline diamond films. Phys. Rev. B. 1989. 40. P. 1817; doi: 10.1103/PhysRevB.40.1817.

\section{Authors and CV}

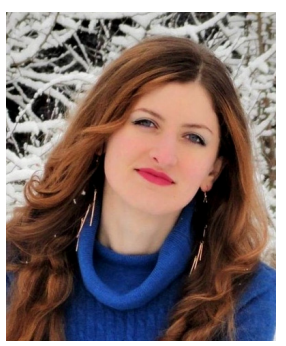

Seneta Mariana Yaroslavivna

PhD-student of Drohobych Ivan Franko State Pedagogical University,

Professional interests: The formation of adsorbed superlattices under the action of a surface standing acoustic wave and electronic surface properties;

Pattern recognition using acousto-location.

E-mail: marsen18@i.ua

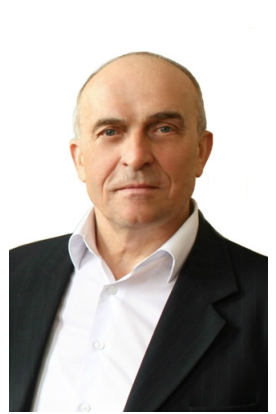

Peleshchak Roman

Mychailovych Head of the Depacrtment of Physics of Drohobych Ivan Franko State Pedagogical University

Doctor of Physical and Mathematical Sciences, Professor, Excellence of Education of Ukraine.

Peleshchak Roman received his degree of Doctor of Physical and Mathematical Sciences in Ivan Franko National University of Lviv in 2001. The theme of dissertation is "Electron-deformation effects in crystals with structural heterogeneities and in stressed heterosystems". He is the author of more than 320 scientific and educationallymethodical works.

E-mail: rpeleshchak@ukr.net

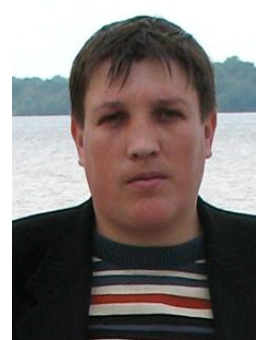

Brytan Victor Bohdanovych

Candidate of Physical and Mathematical Sciences, Associate Professor of Drohobych Ivan Franko State Pedagogical University

Brytan Victor received his degree of Candidate of Physical and Mathematical Sciences in the South Ukrainian National Pedagogical University named after K.D. Ushynsky in 2014. The theme of dissertation is "Influence of atomic hydrogen impurities on electrical and optical properties of CdTe and $\mathrm{Cd}_{1-\mathrm{x}} \mathrm{Zn}_{\mathrm{x}} \mathrm{Te}$ monocrystals grown by sublimation method".

E-mail:vbrytan2@gmail.com 